\title{
$\forall$

\section{AVALIAÇÃO DO LEAN SCORE DE UMA EMPRESA COSNTRUTORA DE GOIÂNIA POR MEIO DA FERRAMENTA LEAN CONSTRUCTION ASSESSMENT TOOL (LCAT) ${ }^{1}$}

\author{
AMARAL, Tatiana Gondim do (1); CASTRO, Amanda Arruda (2) \\ (1) Universidade Federal de Goiás, tatianaagondiml@ufg.br (2) Universidade Federal de \\ Goiás, amanda_castro92@yahoo.com.br
}

\begin{abstract}
RESUMO
A construção civil tem observado uma baixa em seus resultados, como consequência da retração econômica vivida nos últimos anos, o que torna estratégico e necessário o aumento do desempenho das construtoras, com igual redução de custos. Este trabalho tem o objetivo de avaliar o resultado lean score de uma construtora goiana e, também, uma possivel interferência da gestão dos engenheiros no grau de implantação da construção enxuta da empresa. A pesquisa foi classificada como estudo de caso, aplicada, exploratória e qualitativoquantitativa. Foi aplicada a ferramenta Lean Construction Assessment Tool (LCAT) em três obras de uma construtora e no escritório de projetos para obter o grau lean de cada uma das obras e, por consequência, da empresa em si. Observou-se uma considerável divergência de lean score entre as obras. A empresa enquadrouse como nível alto de implantação dos princípios lean, com resultado percentual lean score de 75,51\%.
\end{abstract}

Palavras-chave: Produção enxuta, Construção enxuta, Métodos avaliativos, Construção civil, Grau de implementação.

\begin{abstract}
Civil construction has seen a drop in its results, as a consequence of the economic downturn experienced in recent years, which makes it necessary and strategic to increase the performance of the construction companies, with equal cost reduction. This work has the objective of evaluating the lean score of a construction company in Goiás and also a possible interference of the management of the engineers in the degree of implementation of the lean construction of the company. The research was classified as a case study, applied, exploratory and qualitative-quantitative. The Lean Construction Assessment Tool (LCAT) was applied in three sites of a construction company and in the project department to obtain the lean grade of each one of the sites and, consequently, of the company itself. There was a considerable divergence of lean score between the sites. The company, with a $75.51 \%$ lean score percentage, was a high level of implementation of lean principles.
\end{abstract}

Keywords: Lean production, Lean construction, Assessment methods, Construction, Implementation degree.

\section{INTRODUÇÃO}

Diversos autores puderam constatar a falta de pesquisas no sentido de medir e avaliar a implementação da mentalidade enxuta na indústria da construção civil (KIM; PARK, 2006; LORENZON, 2008; WALTER; TUBINO, 2013).

Sabe-se da importância de mensurar o nível de progresso da implementação a fim de obter dados relacionados ao desempenho dos processos, melhores práticas e área que necessitam de melhorias (SUSILAWATI et al., 2015). A falta de trabalhos que buscaram desenvolver

\footnotetext{
${ }^{1}$ AMARAL, T. G. do; CASTRO, A. A. Avaliação do lean score de uma empresa construtora de Goiânia por meio da ferramenta Lean Construction Assessment Tool (LCAT). In: SIMPÓSIO BRASILEIRO DE GESTÃO E ECONOMIA DA CONSTRUÇÃO, 12., 2021, Maceió. Anais[...] Porto Alegre: ANTAC, 2021. p.1-8. Disponível em: https://eventos.antac.org.br/index.php/sibragec/article/view/425. Acesso em: 2 out. 2021.
} 
métodos para a avaliação do grau de implementação da construção enxuta é ainda um entrave para o sucesso e disseminação da construção enxuta.

Na última década alguns autores desempenharam esforços na tentativa de desenvolver um método de avaliação aplicável à construção enxuta, seja a partir de adaptações de métodos propostos para outros ramos, ou pela proposição de formulários desenvolvidos para verificar a eficácia de ferramentas e práticas lean (SILVA et al., 2014; ETGES et al., 2013; HOFACKER et al., 2008; NESENSOHN et al., 2014; NESENSOHN; BRYDE; PASQUIRE, 2015; SALEM et al., 2006; VALENTE et al., 2012).

No entanto, quando comparados a outros métodos desenvolvidos para diferentes tipos de indústrias, percebe-se a clara tendência para a otimização dos métodos de coleta de dados e para a aplicação de ferramentas mais robustas na análise estatística dos dados, destacando a necessidade por parte dos gestores da utilização de ferramentas de fácil aplicação e que apresentem elevado grau de confiabilidade (WALTER; TUBINO, 2013).

Diante do exposto, o objetivo do trabalho aplicar a ferramenta Lean Construction Assessment Tool (LCAT), desenvolvida por Camargo Filho (2017), para diagnosticar o Lean Score na empresa incorporadora e construtora goiana e avaliar a influência dos gestores neste resultado.

O presente trabalho possui como principal contribuição a avaliação do grau de implementação da construção enxuta conciliando o rigor teórico necessário e a utilização de técnicas que reduzam a subjetividade e viés do aplicador e, por consequência, aumentem a confiabilidade dos resultados.

\section{REFERENCIAL TEÓRICO}

Segundo Susilawati et al. (2015), a verificação do grau de implementação é necessária para determinar os dados relacionados ao desempenho dos processos atuais, as melhores práticas e as áreas que precisam de melhorias das atividades lean.

A ferramenta de avaliação da construção enxuta proposta por Salem et al. (2006) foi desenvolvida para quantificar os resultados da implementação dos princípios da construção enxuta em uma empresa construtora localizada no estado de Ohio, Estados Unidos. O desenvolvimento e aplicação da ferramenta foram realizados por dois grupos distintos: os planejadores e os executores. O check list levantava 24 práticas da construção enxuta. A ferramenta avaliava seis técnicas específicas da construção enxuta, sendo elas: sistema last planner, aumento da visualização, reuniões diárias participativas, estudos preliminares, 5S's e qualidade à prova de falhas.

A ferramenta de avaliação proposta por Carvalho (2008) foi baseada nos 11 princípios da construção enxuta propostos por Koskela (1992) e foi avaliava cinco pontos de vista diferentes na empresa (diretoria, engenharia, operários, fornecedores e projetistas). $\mathrm{O}$ questionário permitia a avaliação dos pontos fortes e fracos da empresa frente à percepção do cliente, possibilitando a busca por melhorias eficientes e estratégicas, ou seja, ações focadas na real necessidade da empresa.

A aplicação do questionário nas cinco diferentes áreas de uma construtora permitia a avaliação do Lean Score da empresa. A avaliação do cliente complementava esse resultado, de forma a direcionar as ações de melhoria da empresa a suprir as falhas percebidas pelo cliente, tendo como consequência um aumento gradual da percepção de valor pelo mesmo. A estrutura do questionário foi composta por perguntas fechadas, elaboradas para avaliar cada um dos 11 princípios propostos por Koskela (1992).

A ferramenta desenvolvida por Hofacker et al. (2008) para aplicação em obras no Brasil e na Alemanha foi denominada Rapid Lean Construction-Quality Rating Model (LCR), entendida 
como um modelo único e fácil de avaliar a qualidade e o grau lean relacionado à busca da filosofia enxuta num projeto de construção. De forma a visualizar e interpretar facilmente os resultados, o índice lean foi classificado de projetos tipo D (que não possuem conhecimento da construção enxuta, baixa aplicação de ferramentas, baixa qualidade e grandes desperdícios) a projetos tipo AAA (alto nível). O questionário foi dividido em grandes categorias, sendo elas: foco no cliente, consciência de desperdício, qualidade, fluxo de material, melhoria contínua e organização, planejamento e fluxo de informações. Juntas, essas categorias somavam 30 perguntas com igual atribuição de peso, ou seja, mesma importância. As notas eram atribuídas de 0 (muito baixo ou inexistente) a 6 (muito alto ou totalmente aplicado). $\mathrm{O}$ resultado final era o percentual de pontos obtidos em relação ao total (180 pontos).

A ferramenta proposta por Valente et al. (2012) foi desenvolvida especificamente para uma construtora brasileira, por meio de um estudo de caso. O checklist foi baseado na ferramenta desenvolvida por Carvalho (2008), nos 11 princípios de Koskela (1992) e nos princípios da produção enxuta desenvolvidos por Womack et al. (1990). O questionário foi dividido em sete diferentes partes: Planejamento e gerenciamento de produção, Kanbans, Jidoka, Fluxos, Produção, Transparência e Limpeza, organização e segurança. As avaliações eram tanto quantitativas quanto qualitativas, e quanto às notas, a atribuição segue os seguintes critérios: 0 (nenhum), 1 (pobre), 2 (bom), 3 (excelente) e não aplicável (devido a cada estágio da obra). $\mathrm{O}$ resultado percentual era alcançado a partir da relação entre a pontuação obtida e a pontuação máxima possível em cada caso.

Etges (2012) desenvolveu um protocolo de auditoria das práticas da construção enxuta, trazendo como novidade a atribuição de fatores de ponderação aos diferentes critérios avaliativos de acordo com a percepção de especialistas da área (mestres e doutores da construção enxuta com publicações de nível internacional). O questionário foi subdividido em 15 categorias, as quais somam 103 práticas a serem observadas, cada uma com seu respectivo conjunto de fontes de evidência. As notas a serem atribuídas eram 0 (não aplicado), 0,5 (parcialmente aplicado) e 1 (totalmente aplicado).

Silva et al. (2014), desenvolveram a ferramenta de avaliação que utiliza a lógica fuzzy, baseada no Fuzzy Interference System (FIS), para diagnosticar e guiar empresas construtoras na transição entre o pensamento tradicional e o pensamento enxuto. O questionário de avaliação foi baseado e dividido de acordo com os 11 princípios de Koskela (1992), num total de 55 aspectos a serem observados. A avaliação era realizada após treinamento do entrevistador, que por meio da entrevista obtém os seguintes dados de entrada: tempo de entrada decorrido entre o primeiro diagnóstico e os seguintes (X1) e nível da presença de uma determinada prática no contexto (X2). O valor de saída (Y) variava de 0 a $10 \mathrm{em}$ uma escala denominada Global Lean Score, permitindo assim que os resultados sejam comparados aos de outras empresas.

A ferramenta desenvolvida por Camargo Filho (2017) denominada Lean Construction Assessment Tool (LCAT) foi baseada nas ferramentas de avaliação já existentes e possuía como proposta a melhoria das lacunas existentes nas demais ferramentas. O questionário foi subdividido em quatro categorias, que representam as diferentes áreas da construção enxuta: Gestão da Qualidade, Gestão da Cadeia de Suprimentos, Planejamento e Controle da Produção e Gestão de Projetos.

A aplicação de cada uma das categorias do questionário em uma empresa foi determinada pelo atendimento ao pré-requisito inerente a esta categoria. Não sendo atendido o prérequisito, a mesma não será avaliada. Cada categoria possuía de 4 a 7 critérios a serem avaliados, cada um possuindo uma série de questionamentos, somando um total de 84 
práticas. Para que estas fossem positivamente contabilizadas, fez-se necessária a verificação das fontes de evidência já previamente determinadas por Camargo Filho (2017).

Quanto às notas, era atribuído o valor 0 quando o item for considerado como não aplicado (evidências de aplicação não encontradas), 1 quando parcialmente aplicado (algumas evidências do Manual de Aplicação encontradas) e 2 quando totalmente aplicado (todas as evidências forem encontradas). Cada item de avaliação possuía um fator de ponderação definido e cada critério possuía um peso em relação ao questionário como um todo para a tabulação das notas.

\section{MÉTODO}

\subsection{Classificação e etapas da pesquisa}

A pesquisa foi classificada como estudo de caso, aplicada, exploratória e qualitativoquantitativa e respeitou as seguintes etapas detalhadas a seguir:

\subsection{Critérios de seleção da empresa participante}

Selecionou-se uma empresa possuía um número considerável de obras em andamento e projetos em fase de lançamento, sendo considerada atuante no mercado, capaz de fornecer resultados consistentes com relação à implementação lean e que poderia absorver as melhorias propostas por esta pesquisa.

\subsection{Caracterização da empresa}

A empresa escolhida atua como construtora e incorporadora no mercado de construções de alto padrão há aproximadamente 11 anos, possuindo mais de 20 empreendimentos concluídos e entregues na cidade de Goiânia (onde está instalada sua sede).

A empresa aplica a cinco anos algumas ferramentas tais como: o Andon, o Kanban, a Linha de Balanço gerenciamento visual, Programa 5's, reuniões de equipe e sistema de prevenção de erros. E busca em seus treinamentos repassar informações quanto a importância da transparência de processos, redução de variabilidade.

Na época da avaliação, a empresa possuía cinco empreendimentos residenciais em construção própria, nas mais diversas fases de obra, nas cidades de Goiânia, Anápolis e Palmas. A empresa também contava com empreendimentos em construção na qual atuava somente como incorporadora, os quais não foram considerados para efeito do presente trabalho. O LCAT foi aplicado às obras em execução na cidade de Goiânia, totalizando três empreendimentos.

\subsection{Caracterização dos empreendimentos}

Foram avaliados três dos cinco empreendimentos em construção da empresa, aqui denominados de A, B e $\mathrm{C}$ em diferentes fases de obra. O Quadro 01 apresenta as características de cada um desses empreendimentos.

Quadro 01 - Caracterização dos empreendimentos participantes

\begin{tabular}{|l|c|c|c|}
\hline & A & B & C \\
\hline Localização & Goiânia & Goiânia & Reiânia \\
\hline Tipologia & Residencial & Residencial & Acabamentos \\
\hline Fase de construção & Estrutura & Acabamentos & $1.810,63 \mathrm{~m}^{2}$ \\
\hline Área do terreno & $1.466,04 \mathrm{~m}^{\mathbf{2}}$ & $1.485,64 \mathrm{~m}^{2}$ & 03 por andar + duas \\
\hline $\mathbf{N}^{\mathbf{0}}$ de apartamentos por andar & 02 (unidades simplex \\
e duplex) & 01 & 89 \\
\hline $\mathbf{N}^{\mathbf{0}}$ de apartamentos totais & 43 & 35 & \\
\hline
\end{tabular}




\begin{tabular}{|l|c|c|c|}
\hline $\begin{array}{l}\text { Percentual de conclusão da } \\
\text { obra }\end{array}$ & $49,56 \%$ & $87,80 \%$ & $81,50 \%$ \\
\hline $\mathbf{N}^{\mathbf{0}}$ de funcionários próprios & 39 & 34 & 46 \\
\hline $\mathbf{N}^{\mathbf{0}}$ de funcionários terceirizados & 66 & 99 & 80 \\
\hline
\end{tabular}

Fonte: Autoria própria.

\subsection{Caracterização dos gestores}

Os gestores de cada empreendimento foram avaliados quanto aos aspectos de formação escolar e experiência profissional, tendo como resultado as informações constantes no Quadro 04. Os gestores foram nomeados conforme a obra sob sua responsabilidade.

Quadro 02 - Caracterização dos gestores dos empreendimentos

\begin{tabular}{|c|c|c|c|}
\hline & Gestor A & Gestor B & Gestor C \\
\hline Idade & 26 & 31 & 32 \\
\hline Instituição de ensino & PUC-GO & PUC-GO & Faculdade Objetivo \\
\hline $\begin{array}{lll}\begin{array}{l}\text { Tempo decorrido } \\
\text { graduação }\end{array} & \text { após } \\
\end{array}$ & Quatro anos & Sete anos & Doze anos \\
\hline $\begin{array}{l}\text { Formação } \\
\text { complementar }\end{array}$ & Não possui & Não possui & MBA - FGV \\
\hline $\begin{array}{l}\text { Área da formação } \\
\text { complementar }\end{array}$ & Não possui & Não possui & $\begin{array}{l}\text { Gerenciamento de } \\
\text { Projetos } \\
\end{array}$ \\
\hline Conhecimentos específicos & $\begin{array}{c}\text { Cursos de Direção, } \\
\text { gestão e fiscalização de } \\
\text { obras e Gestão de } \\
\text { empreendimentos de } \\
\text { engenharia }\end{array}$ & $\begin{array}{l}\text { Cursos de Tecnólogo, } \\
\text { AutoCad e PBQP-h }\end{array}$ & $\begin{array}{c}\text { Cursos de MS Project, } \\
\text { Patologias e Liderança } \\
\text { e Gestão }\end{array}$ \\
\hline Tempo na empresa & Quatro anos & Três anos & Cinco anos \\
\hline
\end{tabular}

Fonte: Autoria própria.

\subsection{Aplicação do questionário}

O LCAT foi aplicado nas três obras supracitadas da empresa, com a participação do avaliador externo e o Engenheiro Responsável. No escritório central da empresa, a avaliação foi feita com o Analista de Projetos. Nas obras foram avaliadas as categorias de Gestão da Qualidade, Gestão da Cadeia de Suprimentos e Planejamento e Controle da Produção do questionário. Os itens de Gestão de Projetos foram avaliados pelas obras e pelo escritório de projeto da empresa.

Com relação à quantidade de itens abordados, 73 dos 84 itens (86,9\% do questionário) foram igualmente aplicados em cada uma das obras, enquanto os 11 itens restantes $(13,1 \%$ do questionário) foram aplicados no escritório de projetos da empresa, em razão de os mesmos não terem sido respondidos em obra por serem considerados, pelos respectivos gestores, como muito específicos da área de projetos da empresa. As notas foram dadas em concordância com as informações cedidas pelos entrevistados, sendo empregado 0 quando o item foi considerado como não aplicado, 1 quando parcialmente aplicado e 2 quando totalmente aplicado.

\subsubsection{Análise dos resultados}

As notas obtidas pela entrevista foram tabuladas por meio da própria ferramenta LCAT, em que cada item de avaliação observado continha um fator de ponderação definido pelo formulário. A partir da multiplicação da nota atribuída aos itens pelo seu respectivo peso, foram somados os resultados dos itens observados, feita a média e multiplicado por uma ponderação definida para cada critério com relação ao questionário total. 
O resultado em percentual de cada categoria foi alcançado pela relação entre a pontuação obtida e a máxima possível para os critérios abordados, sendo o resultado final da obra uma média das porcentagens de cada categoria. Por sua vez, o resultado final da empresa foi a média dos resultados de cada obra. O resultado numérico final da empresa pôde ser comparado ao Lean Score, definido por Camargo Filho (2017).

\section{RESULTADOS E DISCUSSÕES}

Para facilitar a visualização e interpretação dos resultados obtidos, foi elaborado o Quadro 03, no qual é possível analisar os resultados globais em percentuais de cada obra, levando-se em conta as quatro categorias analisadas e o Lean Score de cada obra e da empresa em si.

\begin{tabular}{|c|c|c|c|c|}
\hline Categoria & Obra A & Obra B & Obra C & Empresa \\
\hline Gestão da Qualidade & $72,29 \%$ & $94,81 \%$ & $91,34 \%$ & $86,15 \%$ \\
\hline Gestão da Cadeia de Suprimentos & $68,03 \%$ & $76,68 \%$ & $76,92 \%$ & $73,88 \%$ \\
\hline $\begin{array}{l}\begin{array}{l}\text { Planejamento e Controle da } \\
\text { Produção }\end{array} \\
\end{array}$ & $46,11 \%$ & $45,16 \%$ & $57,90 \%$ & $49,72 \%$ \\
\hline Gestão de Projetos & $84,62 \%$ & $92,31 \%$ & $100,00 \%$ & $92,31 \%$ \\
\hline Lean Score & $67,76 \%$ & $77,24 \%$ & $81,54 \%$ & $75,51 \%$ \\
\hline $\begin{array}{l}\text { Lean Score - faixas propostas por } \\
\text { Camargo Filho }(2017)\end{array}$ & Alto & Alto & Muito Alto & Alto \\
\hline
\end{tabular}

Fonte: Autoria própria.

$\mathrm{Na}$ categoria Planejamento e Controle da Produção, a obra C destacou-se das demais, alcançando 57,90\%. A obra A atingiu o valor de 46,11\%, superior aos 45,16\% da obra B. Entende-se que tais resultados são decorrentes de um maior número de práticas e ferramentas implementadas nos canteiros em decorrência dos conhecimentos específicos aplicados pelos gestores.

Quanto à última categoria a ser analisada, a obra A obteve o menor desempenho entre as três obras analisadas, com $84,62 \%$, embora esta tenha sido a categoria em que obteve seu melhor desempenho. A obra B alcançou o resultado de $92,31 \%$ de adoção das práticas definidas pela ferramenta LCAT, enquanto a obra C obteve $100 \%$ de adesão. Destaca-se que o gestor da obra C possui MBA em Gerenciamento de Projetos, o que poderia contribuir para este resultado.

Foi possível perceber que a empresa obteve como resultado alto grau de implantação da construção enxuta em seus empreendimentos, resultados possíveis por diversas interferências da Comunidade da Construção Civil - GO por meio de palestras e cursos relacionados à temática Lean. $\mathrm{O}$ constante benchmarking entre elas somado à maturidade na implementação do SGQ contribuem com esse resultado.

Embora as obras A e B tenham sido classificadas na mesma faixa de implementação da construção enxuta, pôde-se observar uma considerável diferença em relação às pontuações obtidas: quase 10 pontos percentuais a mais para a obra $\mathrm{B}$. A partir desse primeiro resultado, foi analisado o histórico de gestão de ambas as obras. O gestor da obra A, embora trabalhe na empresa há quatro anos, apenas recentemente havia assumido a gestão da obra em questão. Antes de assumir a gestão da obra, entretanto, o mesmo já trabalhava na obra com o cargo de Analista de Produção, época em que o gestor da obra analisada era o gestor da obra B.

Em relação à obra $\mathrm{B}$, sua gestão passou por um período relativamente instável: até meados de setembro do ano de 2017, com aproximadamente 30 meses de obra, sua gestão já havia sido realizada por seis diferentes engenheiros, mudanças que interferiram significativamente na implantação de princípios e ferramentas. $\mathrm{O}$ atual gestor da obra $\mathrm{B}$ assumiu a mesma em 
setembro de 2017, tendo sido deslocado da obra A para a B. Além disso, a obra passou por três diferentes gerentes de obra.

Com relação à obra $\mathrm{C}$, desde seu início a mesma tem sido gerida pelo gestor $\mathrm{C}$. De fato, dentre os empreendimentos avaliadas foi o que apresentou maior maturidade na implementação, não apenas pelo número de técnicas e ferramentas aplicadas, mas também pelo conhecimento apresentado da teoria da CE. Sabe-se que a simples aplicação de algumas práticas e/ou ferramentas da construção enxuta não implicam na real implementação da filosofia enxuta. Por outro lado, a avaliação da presença de práticas indiretamente possibilita a visualização de indícios da extensão da implementação e disseminação dos princípios na empresa.

Pôde-se observar que a obra A obteve menor desempenho em relação às demais devido tanto à mudança de gestão quanto ao fato de que o novo gestor possui menor tempo de experiência como engenheiro responsável e também na participação de treinamentos com a temática lean. Isso pôde ser comprovado pelo fato de que o gestor B, em sua atual obra, tenha obtido melhor desempenho, adotando maior número de práticas lean.

Acredita-se que a mudança de gestão influencia diretamente na manutenção das práticas do lean construction, pois é um momento de reestruturação da gestão, com consequente diminuição de desempenhos gerais devido ao momento de adaptação do atual gestor.

\section{CONCLUSÕES}

Por meio do LCAT foi possível determinar o Lean Score nos empreendimentos da empresa e perceber o grau de conhecimento dos gestores quanto aos princípios lean. Foi observado que grande parte das ferramentas era corretamente entendida e utilizada pelos gestores, mas sem uniformidade.

Comparando-se as obras B e C foi possível observar que a gestão contínua do gestor C influenciou positivamente em seus resultados e que o mesmo possuía um maior conhecimento acerca da filosofia lean e suas ferramentas de aplicação em relação aos demais.

Como sugestão de futuros trabalhos tem-se a análise da interferência de obras em outras cidades nas práticas adotadas e consequentemente no resultado lean da empresa.

\section{REFERÊNCIAS}

CAMARGO FILHO, C. A. B. LCAT: Ferramenta de Avaliação da Implementação da Construção Enxuta. 2017. 99 f. Monografia (Exame de Qualificação) - Programa de Pós Graduação em Geotecnia, Estruturas e Construção Civil, Universidade Federal de Goiás, Goiás, 2017.

CARVALHO, B. S. Proposta de uma ferramenta de análise e avaliação das construtoras em relação ao uso da construção enxuta. 2008. 141 f. Dissertação (Mestrado em Engenharia Civil) - Curso de Pós-graduação em Construção Civil, Universidade Federal do Paraná, 2008.

ETGES, B. M. B. S. Protocolo de auditoria do uso de práticas da construção enxuta. 2012. 85 f. Tese (Mestrado em Engenharia da Produção) - Escola de Engenharia Civil, Universidade Federal do Rio Grande do Sul, Porto Alegre, 2012.

ETGES, B. M. B. S.; SAURIN, T. A.; BULHÕES, I. R. Identifying Lean Construction Categories of Practices in the IGLC Proceedings. In: 20TH ANNUAL CONFERENCE OF THE INTERNATIONAL GROUP FOR LEAN CONSTRUCTION. 2012Disponível em: <http://www.iglc.net/Papers/Details/767>. Acesso em: 22 set. 2015 
HOFACKER, A.; FERNANDES, B.; GEHBAUER, F. FREITAS, M. C. D.; MENDES, R.; SANTOS, A.; KIRSCH, J. Rapid lean construction - quality rating model. In: 16th Annual Conference of the IGLC, Manchester, UK. Proceedings... Manchester, UK. 2008.

LORENZON, I. A. A medição de desempenho na construção enxuta: estudos de caso. 2008.

KOSKELA, L. Application of the new production philosophy to construction. Stanford Center for Facility Engineering, TECHNICAL REPORT 72, 1992, 75p.

KIM, D.; PARK, H.-S. Innovative construction management method: Assessment of lean construction implementation. KSCE Journal of Civil Engineering, v. 10, n. 6, p. 381-388, nov. 2006.

NESENSOHN, C. et al. Assessing Lean Construction Maturity. . In: 22ND ANNUAL CONFERENCE OF THE INTERNATIONAL GROUP FOR LEAN CONSTRUCTION. 2014Disponível em: <http://www.iglc.net/Papers/Details/981>. Acesso em: 7 out. 2015

NESENSOHN, C.; BRYDE, D.; PASQUIRE, C. A Measurement Model for Lean Construction Maturity. In: 23RD ANNUAL CONFERENCE OF THE INTERNATIONAL GROUP FOR LEAN CONSTRUCTION. 2015Disponível em: <http://iglc.net/Papers/Details/1166>. Acesso em: 1 jun. 2016

SALEM, O.; SOLOMON, J.; GENAIDY, A.; MINKARAH, I. Lean Construction: From Theory to Implementation. Journal of Management in Engineering, v. 22, n. 4, p. 168-175, 2006.

SILVA, R. S. M.; AMARAL, T. G.; SILVA, F. M. A. Fuzzy logic applied to lean construction - An implemetation in building companies. Journal of Civil Engineering and Architecture Research. v. 1, n. 1, p. 37-44, 2014.

SUSILAWATI, A.; TAN, J.; BELL, D.; SARWAR, M. Fuzzy logic based method to measure degree of lean activity in manufacturing industry. Journal of Manufacturing Systems, v. 34, p. $1-11,2015$.

VALENTE, C.; NOVAES, M.; MOURÃO, C. A.; NETO, J. Lean monitoring and evaluation construction site: a proposal of lean audits. In: 20th Annual Conference of the IGLC, San Diego, USA. Proceedings... San Diego, USA. 2012.

WALTER, O. M. F. C.; TUBINO, D. F. Assessment methods of lean manufacturing: literature review and classification. Gestão \&amp; Produção, v. 20, n. 1, p. 23-45, mar. 2013.

WOMACK, J. P.; JONES, D. T.; ROOS, D. The machine that changed the world. Macmillan, New York, USA, 1990. 